\title{
A Reliable and Energy Efficient VBF-improved Cross-layer Protocol for Underwater Acoustic Sensor Network
}

\author{
Ning Sun ${ }^{1}$, Guangjie Han ${ }^{1}$, Tongtong $\mathrm{Wu}^{1}$, Jinfang Jiang ${ }^{1}$, Lei Shu ${ }^{2}$ \\ ${ }^{1}$ College of Internet of Things Engineering, Hohai University, Changzhou, China \\ ${ }^{2}$ Guangdong University of Petrochemical Technology, Maoming, China \\ sunn2001@hotmail.com, hanguangjie@gmail.com, wutong8023@163.com, \\ jiangjinfang1989@gmail.com, lei.shu@ieee.org
}

\begin{abstract}
Underwater sensor networks (USNs) has many characteristics different from terrestrial wireless sensor networks (WSNs), such as dynamic network topology, unreliable acoustic communication, which increases the difficulty in energy efficiency and reliability of data transmission, for traditional WSN protocols are not suitable for underwater acoustic sensor networks (UASNs). Vector based forwarding (VBF) protocol is an energy efficient routing protocol for UASNs, by using the location information of nodes to limit the scale of flooding so that to save energy consumption and handle the mobility of nodes. In this paper a cross-layer protocol is proposed, which not only utilizes the VBF-based routing algorithm, but also considers the residual energy and the times of data relay in a cycle time to make more optimized decision whether a node will forward data or not. According to the simulation results, more evenly energy consumption and reliable data transmission are achieved, compared to previous VBF-based routing protocols for UASNs.
\end{abstract}

Keywords-UASN; cross-layer protocol; VBF; reliability; energy efficiency

\section{INTRODUCTION}

Recently, there have been growing interests in underwater wireless sensor network (UWSN) applications, such as oceanographic data collection, oil exploration, pollution monitoring and tactical surveillance, etc.

Since underwater sensors are usually deployed in threedimensional space and the location of the nodes usually changes with the water flow, the topology of USNs is more complex than terrestrial WSNs. Most of the traditional routing protocols for terrestrial wireless sensor networks (WSNs) [1] are not suitable for USNs. It results in new challenges for designing routing protocols for USNs [2].

Acoustic communication is regarded the most appropriate medium in underwater environment. However, due to the physical characteristics of sound signals, acoustic channels are

The work is supported by 'the Fundamental Research Funds for The Central Universities, No.2014B01714' , 'Natural Science Foundation of JiangSu Province of China, No.BK20140248', 'the Qing Lan Project', 'the Educational Commission of Guangdong Province, China Project No. 2013KJCX0131', 'the Guangdong High-Tech Development Fund No. 2013B010401035', and 'National NSFC Project No. 61401107'. featured with low available bandwidth, large propagation delay and high error probability. End-to-end reliable transmission is an important and difficult issue for designing protocol for UASNs.

Moreover, due to the special nature of the underwater environment, the nodes working in the underwater environment are generally equipped with battery power, which is hard to replace or recharge. Energy issues directly affect the life of the entire sensor network. Above all, it is necessary to design a reliable and energy efficient protocol suitable for UASNs to deal with the problems of complex dynamic topology, unreliable communication channel and energy constraint.

In [3], an energy saved vector-based data forwarding protocol for UWSNs is proposed. The intermediate node decides whether to forward packet or not by weighing the benefits according to its location. By this way, the number of packet-forwarding nodes is decreased and accordingly the energy consumption is reduced. The packets are forwarded in redundant and interleaved paths, which add reliability of data transmission. However, only location factor is considered as weighing the benefit of forwarding, which easily results in uneven energy consumption between sensor nodes and shortens network lifetime. Furthermore, the reliability of data transmission can't be guaranteed in the situation of sparse network.

VBF and its improved protocols [4][5] are routing protocols, which are only designed for network layer. The medium access problem in conventional UASNs didn't be considered in these works. A number of MAC solutions [6][7] have been proposed for UASNs. Deterministic MAC protocols such as TDMA, FDMA, or CDMA cannot be directly adopted in UASNs due to problems such as narrow channel bandwidth, long propagation delay, difficulty in clock synchronization, etc. Therefore, efficient MAC protocol for UASNs is necessary, at the same time, more difficult to implement in underwater environment than in terrestrial WSNs. According to [8], cross-layer protocol is an efficient solution to deal with the energy and reliability problems in UASNs, which integrates MAC layer, network layer and transport layer functionalities and is carried out more practically than single layer protocol. 
In this paper, we proposed a reliable, scalable and evenly energy-consumed cross-layer protocol for UASNs, which integrates MAC and network layer design. Evenly energy consumption, extended network lifetime and reliable data transmission is the purpose of our proposed protocol. On the basis of VBF [3] protocol, taking both location information and residual energy of nodes into account, evenly energy consumption can be obtained. Furthermore, the times of packet relay of a node in last round time is regarded as a key factor to decide whether this node will join the packet forwarding in this round time, which is able to decrease possible medium collisions and save energy consumption of active intermediate node efficiently. A simple ACK process is carried out in order to increase the end-to-end reliability of data transmission. Global network information and clock synchronization at nodes are not required and the protocol is run in distributed way so that scalability is guaranteed and extra traffic communication is avoided.

The rest of the paper is organized as follows. Routing and MAC protocols for UASNs are introduced in Section II. In Section III, the proposed cross-layer protocol is described in details and some analysis is made. In section VI, simulation works are introduced and the simulation results are analyzed. In Section V, we draw the conclusion.

\section{RELATED WORKS}

\section{A. Location-based Rouring Protocols for UASNS}

The first routing protocol designed for mobile underwater sensor networks is Vector Based Forwarding (VBF) protocol, which was proposed in [3]. VBF is a trajectory-based forwarding protocol. It represents a trajectory with a "routing vector" from the source to the sink. Intuitively a virtual pipe with the source-to-sink vector as the axis is used as the abstract route for data delivery. If the pipe is "populated" by nodes then the data packets can be forwarded to the sink. The radius of the virtual pipe is a predefined distance threshold. For any sensor node which receives data, it first computes its distance to the routing vector. If this distance is smaller than the threshold, then the node is considered as a candidate to forward the data. Otherwise, the node simply discards the data. To reduce the traffic in dense networks, VBF adopts a distributed selfadaptation algorithm, in which all the candidate nodes are coordinated and finally only several most "desirable" ones can forward the data packets. Compared with naive flooding, VBF can significantly reduce network traffic, thus saving energy. It is also robust to topology dynamics since it is a location-based on-demand routing protocol, and no pre-computed routes maintained in sensor nodes.

One of VBF-improved protocol is the Hop-by-Hop VectorBased Forwarding (HH-VBF) [4], which pointed that, by using the unique source-to-sink vector, the creation of a single virtual pipe may significantly affect the routing efficiency in different node density areas. Meanwhile, VBF is too sensitive to the routing pipe radius threshold. To overcome these problems in VBF, It uses the same concept of routing vector as VBF. However, instead of using a single virtual pipe from the source to the sink, HH-VBF defines a different virtual pipe around the per-hop vector from each forwarder to the sink. In this way, each node can adaptively make packet forwarding decisions based on its current location. However, under conditions of densely distributed nodes, HH-VBF protocol does not effectively limit the broadcast range of data, resulting in unnecessary energy consumption.

The other one is proposed in [5], which is the improvement of VBF protocol. Both the location and energy status of node is considered when deciding whether to forward packet, which reduces the imbalanced energy consumption. Acknowledgement of receiver is used to improve the reliability of data transmission, at the same time reduce redundant data forwarding. However, the underwater node is mobile so there may be a plurality of data sources in this area. This will lead to a problem: the node A, whose current residual energy is few, will not use again in the next period of time probably, while the node $\mathrm{B}$ with more residual energy just at a key position of a plurality of pipes. This is likely to result in high-frequency use of $B$ in a certain period of time. Furthermore, it results in premature death of key nodes, thereby reducing the success rate of data transmission.

[6] is a multi-path routing protocol using location information for UASNs. Reliability of data transmission is improved efficiently. However, network delay and energy efficiency is paid as the cost.

\section{B. MAC Protocols for UASNs}

A number of solutions have been proposed for the medium access problem in conventional underwater acoustic sensor networks. Deterministic MAC schemes such as TDMA, FDMA, or CDMA cannot be directly adopted in the underwater environment due to problems such as narrow channel bandwidth, vulnerability to fading and multipath, dependence on network-wide clock synchronization, handling long propagation delays, optimizing energy consumption, difficulty of power control at each node to avoid the near-far problem, and scalability with number of nodes [8].

The handshaking protocol in [10] requires synchronization of all nodes. A node is allowed to reserve multiple time slots for transmission, and the scheduled transmission cannot be canceled even if another communicating neighbor is detected. In [11], an improved RTS/CTS handshaking solution that does not need clock synchronization is proposed. RTS/CTS handshaking brings extra delay to MAC operation. The protocol assumes that all nodes use the same transmission power, which is not valid for UASN. To solve any uncertainty, coordination of medium access is carried out by a centralized controller in [12]; however, deciding on a network-wide collision-free transmission order requires knowledge of relative locations of all nodes. It is also proposed to choose a transmission order at the receiver side [13]. The receiver has to wait until it receives an RTS from all possible contenders, and this decreases channel utilization seriously. [14] combines carrier sensing with a packet exchange between sender and receiver before transmission. Nodes rely on global time synchronization, and a node is allowed to transmit only at the beginning of a time slot. 


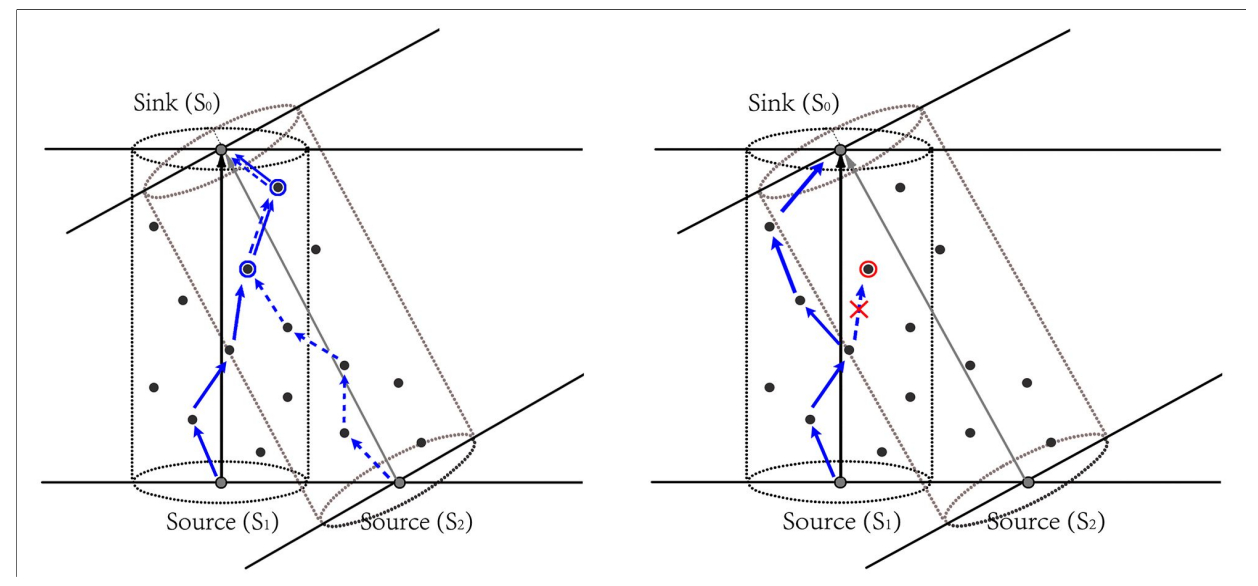

Figure 1. a) VBF-based protocol without consideration of the times of packet relay in cycle time; b) protocol with consideration of the times of packet relay in cycle time

Reservation-based medium access is another design alternative [15]-[18]. Nodes estimate propagation delays for scheduling transmissions in [15]. However, this estimation is more applicable when nodes are static and no new node joins the network. [16] is a MAC solution for single-hop underwater networks, where each node has to count how many other nodes contend for the channel. [17] is a TDMA implementation, requiring synchronization of transmission schedules through periodic message exchange. Similarly, in [18], receivers periodically start packet transfers, generating continuous packet exchange overhead in the network. Furthermore, for a dynamic topology under channel fluctuations, it is not very practical to determine when to initiate transmission at each node.

\section{THE Proposed CROSS-LAYER PROTOCOL}

In this section, we introduce a cross-layer protocol for UASNs, which is reliable and energy evenly consumed, and analyze the improvements of the proposed protocol compared with VBF protocol.

\section{A. Network Model}

USNs work naturally in a three-dimension and mobile environment. It is assumed that sensor nodes are deployed in layers of different depths. At each layer, nodes can float with water current. The sink node is usually located in the surface of water. Data is delivered from nodes underwater to sink node in surface of water. The nodes are mobile due to the movement with water current, so routing protocol should be designed to adapt the dynamitic network topology. Here, we assume the network is dense enough that each node is in the range of transmission of at least one neighbor. It is also resumed that each node knows its residual energy and location information.

\section{B. Overview of the Protocol}

As shown in [3], the data forwarding path is specified by the routing vector from source node to destination (generally sink node). Forwarders along the routing vector form a routing pipe with a predefined radius $W$. Nodes resided within the pipe have opportunity to forward packet. It limits the area of data flooding such that energy consumption is saved. However, the vector-based forwarding algorithm only considers the location information of the node when deciding whether the node takes part in data forwarding. In situation of multiple source nodes, VBF protocol easily causes unevenly energy consumption between nodes. For instance, in Figure 1, some nodes are in both packet forwarding paths, respectively from source $S_{1}$ and source $\mathrm{S}_{2}$. They need to do relay works more frequently than other nodes, which results in quick energy exhaustion of them if using VBF algorithm. Moreover, when the nodes lay in two different paths, there is problem of medium collision, which could lead to unreliable data transmission. Therefore, finding the joint nodes laying in different packet forwarding paths and avoiding (or reducing) using them in next round is an efficient cross-layer method to balance energy consumption and improve reliable end-to-end data transmission.

In this protocol, we redefined the policy of selection of forwarding nodes. Instead of only using the position information as a forwarding node factor, residual energy of nodes and the times of packet relay in last round is also taken into accounts as the data forwarding factor.

\section{Operation Process of the Protocol}

The proposed protocol contains two basic packet type, the one is query packet (Q-Packet), and the other one is data packet (D-Packet).Q-Packet includes packet information (PID, $S P, T P$, $F P, \quad W, R, A . R$-Energy, A.N), where $S P$ means the source position, $F P$ means the position of forwarding position, $T P$ is the target position, generally, the target is the sink node, $R$ is a given transmission radius and $W$ is the radius of the routing vector. EEC-VBF assumes that node $\mathrm{A}$ is the current forwarding node and $\mathrm{B}$ is one of the candidate nodes, and A.REnergy means the residual energy of $\mathrm{A}$ and $A . N$ means the times of participation in data forwarding by A in last round. DPacket contains the data to be transmitted, additionally, the packet head includes the information of the set of next forwarding nodes specified by A.

When a Q-Packet received by a candidate node named B, the node will first judge the effectiveness of the package by $P I D$. If $\mathrm{B}$ has not forwarded the package, it will compute the distance from $\mathrm{A}$ to $\mathrm{B}$ and determine the relative position of $\mathrm{B}$. The desirableness factor (DF) is calculated as the following: 
$\alpha=k_{1} \times\left(\frac{A \cdot \text { Renergy }}{B \cdot \text { Renergy }}\right)+k_{2} \times\left(\frac{p}{W}+\frac{(R-d \times \cos (\theta))}{R}\right)^{2}+k_{3} \times\left(\frac{A \cdot N}{B \cdot N}\right)$

where $p$ is the projection of $\mathrm{B}$ to the routing vector, $W$ is the radius of routing pipe, $R$ is the transmission range, $d$ is the distance between $\mathrm{A}$ and $\mathrm{B}$, and $\theta$ is the angle between vector $\mathrm{AD}$ and vector $\mathrm{AB}$, whereas A.Renergy is the residual energy of A and B.Renergy is for B. A.N refers to the times of packet relay in last round by $\mathrm{A}$ and $B . N$ refers to by B. $k_{1}, k_{2}$ and $k_{3}$ are the weight value for three factors, which can be adaptive according to application requirements.

Since then, node $\mathrm{B}$ will hold the packet for a time interval ( $\mathrm{T}_{\text {adaption }}$ ) proportional to its desirableness factor $(\alpha)$.

$T_{\text {adaption }}=\sqrt{\alpha} \times T_{\text {delay }}+\frac{(R-d)}{v}$

Therefore, the node with lest desirableness factor will send the acknowledgement message (ACK) to A. This process is described in detail in ALGORITHM B_1 as follow:

\begin{tabular}{c}
\hline ALGORITHM B_1: Algorithm for node selections and packet \\
forwarding
\end{tabular}

1: Get packet information (PID, SP, TP, FP, W, R, A.R-

Energy, A.N) from Q-Packet

2: IF(check $P I D$, and the packet has been received)

3: Drop packet

4: ELSE

5: Compute the distance from the previous node to this node (d);

6: Compute the position of $\mathrm{B}$;

7: Compute the distance $(p)$ from $\mathrm{B}$ to its projection on

8: Compute desirableness factor as

$$
\alpha=k_{1} \times\left(\frac{A \cdot \text { Renergy }}{B \cdot \text { Renergy }}\right)+k_{2} \times\left(\frac{p}{W}+\frac{(R-d \times \cos (\theta))}{R}\right)^{2}+k_{3} \times\left(\frac{A \cdot N}{B \cdot N}\right)
$$

9: Compute $\mathrm{T}_{\text {adaption }}$ as

$$
T_{\text {adaption }}=\sqrt{\alpha} \times T_{\text {delay }}+\frac{(R-d)}{v}
$$

10: Wait for a period of time specified by $\mathrm{T}_{\text {adaption; }}$

11: Send ACK message to A;

12: END IF

Furthermore, A will specify the nodes which are going to forwarding the D-Packet and write the information of these nodes into the header of D-Packet. The node B, which is one of the next forwarding nodes, will update the header of Q-Packet after receiving D-Packet successfully. First of all, Node B will be read in its own cycle forwarding number $B . N$ and incremented by one, then put it as a parameter along with $B . R$ Energy to be written to the Q-Packet and overwrite the original data. Furthermore, the node B will send the Q-Packet and do the next hop forwarding. After packet forwarding, node will check timer, then clear $N$ and resume counting if the running time has more than one cycle.

This process is described in detail in ALGORITHM B_2 as follow:
ALGORITHM B_2: Algorithm for node selections and packet forwarding

1: Get ID SET from D-Packet;

2: $\operatorname{IF}(B . I D$ is not in ID SET)

3: Drop packet;

4: ELSE

5: $\quad$ Update $B . N=B . N+1$;

6: $\quad$ Update B.R-Energy;

7: Write B.N, B.R-Energy into new Q-Packet and send it;

8: $\quad$ IF(Get ACK message)

9: Write candidate nodes' ID SET into D-Packet;

10: Send D-Packet;

11: ELSE IF (Over time limit)

12: Drop packet;

13: ELSE;

14: Wait for ACK message;

15: $\quad$ END IF;

16: END IF;

17: $\operatorname{IF}($ Over the set period of time)

18: $\quad B . N=0$;

19: END IF;

20: END IF;

D. ACK Process

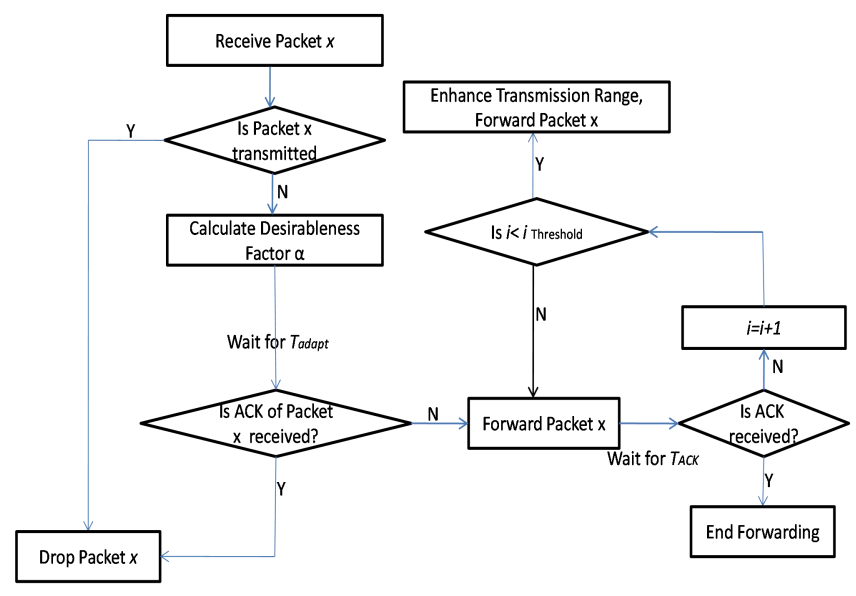

Figure 2. ACK process

A simple ACK process is needed in the proposed protocol. There are two advantages by using ACK process. First, reliability of data transmission is improved. As shown in Figure 2 , if the sender can't receive ACK packet from the next hop receiver after a certain number of times, the sender will enhance its transmission range such that the packet could arrive at the next hop. It is usually efficient in sparse network environment. Secondly, the repeated data can be dropped in time when the sender finds its next hop node has received the same packet. Though network delay and some extra energy consumption for transmission of control packets have to be paid, obvious improvement of end-to-end data transmission reliability can be achieved. 


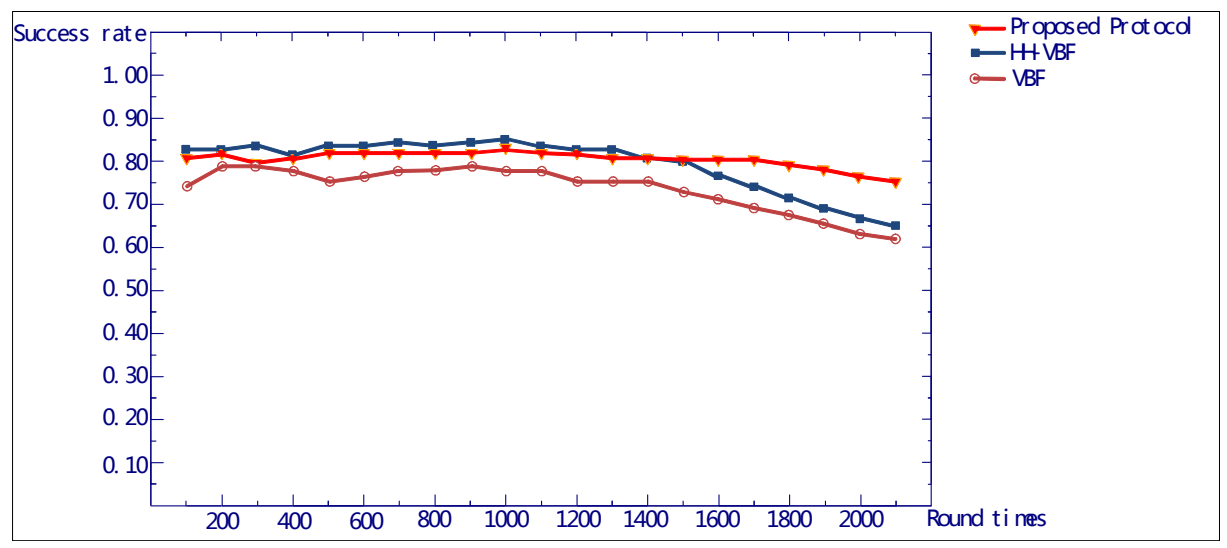

Figure 3. Results of successful rate of data transmsion

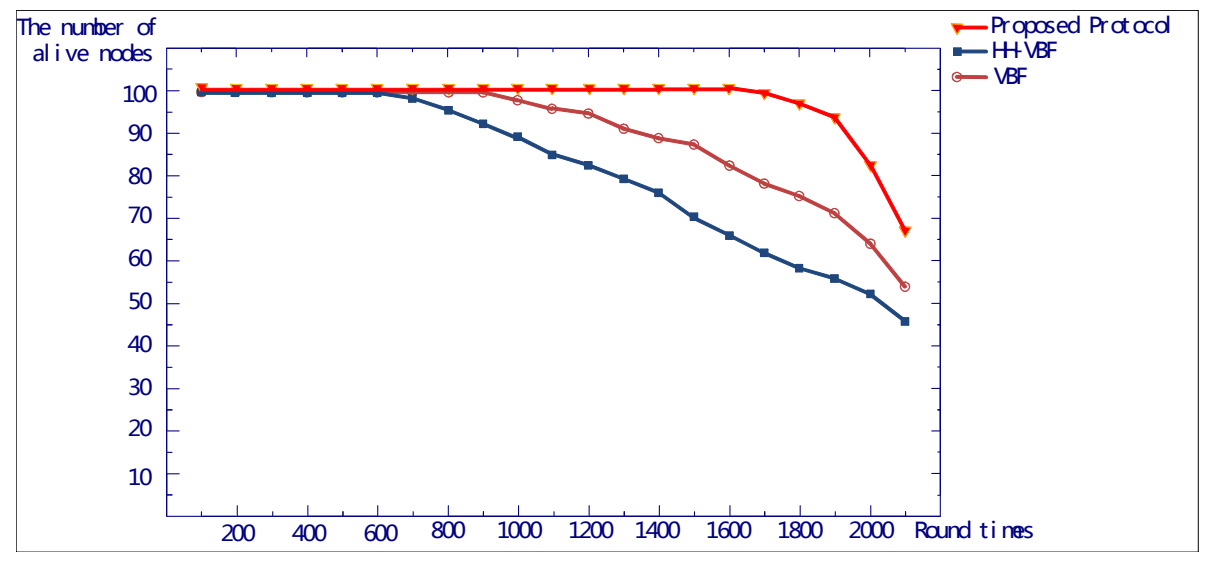

Figure 4. Results of network lifetime

\section{SIMULATION}

In this section, the performance of the proposed protocol is evaluated in terms of network lifetime and reliability and compared with $\mathrm{VBF}[3]$ and $\mathrm{HH}-\mathrm{VBF}[4]$.

\section{A. Simulation Setting}

The simulation is performed using Java 8 and we simulate the underwater environment for three-dimensional network deployment. Table 1 shows the simulation parameters. We use Urick propagation model [19] to describe the transmission loss and use the equation described in [20] to calculate the propagation energy consumption.

TABLE I. SIMULATION PARAMETERS

\begin{tabular}{|c|l|}
\hline Space & $1000 \mathrm{~m} * 1000 \mathrm{~m} * 500 \mathrm{~m}$ \\
\hline Node Number & 200 \\
\hline Sending interval & $1 \mathrm{~s}$ \\
\hline Sink node & $(500,500,500)$ \\
\hline Node speed & random value between $0 \mathrm{~m} / \mathrm{s}-5 \mathrm{~m} / \mathrm{s}$ \\
\hline Position Update & $0.3 \mathrm{~s}$ \\
\hline Vector radius & $100 \mathrm{~m}$ \\
\hline $\begin{array}{c}\text { Transmission } \\
\text { radius }\end{array}$ & $100 \mathrm{~m}$ \\
\hline Source node & Random \\
\hline
\end{tabular}

\section{B. Performance Analysis}

Based on the simulation results, we analyze two aspects. Reliability is measured by successful rate of end-to-end data transmission, while network lifetime is evaluated by number of nodes alive with round time.

\section{1) Reliability of Data Transmission}

The success rate of data transmission of three routing protocols at different time is shown in Figure 3. The data packet received rate of three routing protocols decreases with over time, however the proposed protocol, compared to the other protocols, can received more packets. During the round $100-2000$, the packet received rate of proposed protocol is always higher than VBF. During the round 100-1500, the success rate of proposed protocol is similar to the rate of HHVBF nearly, but after the round 1500, proposed protocol received more packet than $\mathrm{HH}-\mathrm{VBF}$ obviously. As can be seen from Figure 3, after the round 2000, the average success rate of $\mathrm{HH}-\mathrm{VBF}$ is only $67 \%$, while the average success rate of proposed protocol remains at $75 \%$. Because $\mathrm{HH}-\mathrm{VBF}$ and VBF choose the next hop nodes not depending on the residual energy and the possible energy consumption trends of candidate nodes, the energy of part of nodes is depleted and premature death with the increase of the number of rounds, which lead the number of normal node declines greatly and shorten the stabilization period, reducing the ratio of received packets. The proposed protocol adopts strategies to protect the low energy nodes and the key nodes in forwarding process, so 
as to achieve the effect of evenly energy consumption and prolong the survival of the nodes. The Figure 4 also proved this point.

\section{2) Network Lifetime}

Figure 4 illustrates the relationship between the number of surviving nodes and round times. As shown in Figure 4, the number of nodes alive of three protocols decreases as round number increases, but $\mathrm{HH}-\mathrm{VBF}$ is the earliest one which appears the first dead node after the round 600. It is not difficult to understand, without the consideration of residual energy, the ratio of received packets is improved by making more nodes involved into forwarding process. This strategy does not effectively limit the broadcast range of data, resulting in unnecessary energy consumption. In contrast, VBF protocol is better than HH-VBF protocol in this respect, in which first dead node appears after the round 900. However, taking the residual energy and the possible energy consumption trends of candidate nodes into consideration, the proposed protocol still maintains a high reliability even after the round 1600 . Although most of the residual energy has been failure at this point and lead the number of normal node declines greatly, from Figure 4 and Figure 4 we can see that the proposed protocol has obvious advantages in balancing node energy consumption and ensure high reliability of data transmission.

\section{CONCLUSION}

A VBF-improved cross-layer protocol for underwater acoustic sensor networks is proposed in this paper, which uses both the location information and residual energy of intermediate nodes to make decision of data forwarding. Additionally, the times of packet relay by node in a cycle time is taken into account as the factor to decide whether the node is in the data transmission path. Compared to previous vectorbased routing protocols, evenly energy consumption and more reliable data transmission are achieved according to the simulation performance.

\section{REFERENCES}

[1] Prathap, U., et al. "Wireless sensor networks applications and routing protocols: survey and research challenges." Cloud and Services Computing (ISCOS), 2012 International Symposium on. IEEE, 2012.

[2] Ayaz, Muhammad, et al. "A survey on routing techniques in underwater wireless sensor networks." Journal of Network and Computer Applications 34.6 (2011): 1908-1927.

[3] Xie, Peng, J. H. Cui, and L. Lao. "VBF: vector-based forwarding protocol for underwater sensor networks." Proceedings of the 5th international IFIP-TC6 conference on Networking Technologies, Services, and Protocols; Performance of Computer and Communication
Networks; Mobile and Wireless Communications Systems SpringerVerlag, 2006:1216--1221.

[4] Nicolaou, N., et al. "Improving the Robustness of Location-Based Routing for Underwater Sensor Networks." Proc Oceans' (2007):1 - 6.

[5] Wei, Bo, et al. "ES-VBF: An Energy Saving Routing Protocol." Lecture Notes in Electrical Engineering (2013):87-97.

[6] Jinfang Jiang, Guangjie Han, Hui Guo, Lei Shu, Joel J.P.C Rodrigues. Geographic Multi-path Routing based on Geospatial Division in Dutycycled Underwater Acoustic Sensor Networks, Journal of Network and Computer Applications. 2015.

[7] C. Petrioli, R. Petroccia, and M. Stojanovic, "A comparative performance evaluation of MAC protocols for underwater sensor networks," in Proc. OCEANS 2008, pp. 1-10, Sep. 2008.

[8] P. Casari and M. Zorzi, "Protocol design issues in underwater acoustic networks," Computer Communications (Elsevier), vol. 34, no. 17, pp.2013-2025, Nov. 2011.

[9] A. Bereketli, H. Turken, and S. Bilgen, "Cross layer power adaptive CSMA/CA for sink powered underwater acoustic sensor networks", in Proc. MED-HOC-NET(2013), pp.26-33, 2013.

[10] X. Guo, M. R. Frater, and M. J. Ryan, "Design of a Propagation-DelayTolerant MAC Protocol for Underwater Acoustic Sensor Networks,"IEEE Journal of Oceanic Engineering, vol. 34, no. 2, pp. 170-180, Apr. 2009.

[11] B. Peleato and M. Stojanovic, "Distance Aware Collision Avoidance Protocol for Ad-Hoc Underwater Acoustic Sensor Networks," IEEE Communications Letters, vol. 11, no. 12, pp. 1025-1027, Dec. 2007.

[12] Y. J. Chen and H. L. Wang, "Ordered CSMA: a collision-free MAC protocol for underwater acoustic networks," in Proc. OCEANS 2007, pp. 1-6, 2007.

[13] W.-H. Liao and C.-C. Huang, "SF-MAC: A Spatially Fair MAC Protocol for Underwater Acoustic Sensor Networks," IEEE Sensors Journal, vol. 12, no. 6, pp. 1686-1694, June 2012.

[14] M. Molins and M. Stojanovic, "Slotted FAMA: a MAC protocol for underwater acoustic networks," in Proc. OCEANS 2006 - Asia Pacific, pp. 1-7, May 2006.

[15] P. Xie and J.-H. Cui, "R-MAC: An Energy-Efficient MAC Protocol for Underwater Sensor Networks," in Proc. WASA 2007, pp. 187-198, Aug. 2007.

[16] A. A. Syed, W. Ye, and J. Heidemann, "T-Lohi: A New Class of MAC Protocols for Underwater Acoustic Sensor Networks," in Proc. IEEE INFOCOM 2008, pp. 231-235, Apr. 2008.

[17] M.K. Park and V. Rodoplu, "UWAN-MAC: An Energy-Efficient MAC Protocol for Underwater Acoustic Wireless Sensor Networks," IEEE Journal of Oceanic Engineering, vol. 32, no. 3, pp. 710-720, July 2007.

[18] N. Chirdchoo, W.-S. Soh, and K. C. Chua, "RIPT: A Receiver-initiated Reservation-based Protocol for Underwater Acoustic Networks," IEEE Journal on Selected Areas in Communications, vol. 26, no. 9, pp. 17441753, Dec. 2008.

[19] Lurton, Xavier. An introduction to underwater acoustics: principles and applications. Springer Science \& Business Media, 2002.

[20] Harris III, Albert F., and Michele Zorzi. "Modeling the underwater acoustic channel in ns2." Proceedings of the 2nd international conference on Performance evaluation methodologies and tools. ICST (Institute for Computer Sciences, Social-Informatics and Telecommunications Engineering), 2007.

[21] Guangjie Han, Jinfang Jiang, Lei Shu, Yongjun Xu, Feng Wang. Localization Algorithms of Underwater Wireless Sensor Networks: A Survey. Sensors, Vol.12, No.2, pp: 2026-2061, 2012. 